\title{
Persistence and differential distribution of nonindigenous ascidians in harbors of the Southern California Bight
}

\author{
Charles C. Lambert ${ }^{1,2, *}$, Gretchen Lambert ${ }^{1,2}$ \\ ${ }^{1}$ University of Washington, Friday Harbor Laboratories, 620 University Road, Friday Harbor, Washington 98250, USA \\ ${ }^{2}$ Present address: 12001 11th Ave NW, Seattle, Washington 98177, USA
}

\begin{abstract}
The Southern California Bight covers a region of about $300 \mathrm{~km}$ of coastline from northern Baja California to Point Conception. The present study includes ascidian species abundance data from 1994 to 2000 from 29 sites within the 12 major harbors of the Bight north of the Mexico/US border, plus 2 sites in Ensenada (Baja California, Mexico) sampled in August 2000. Nonindigenous ascidians form a major part of the fouling community biomass on floating docks in these harbors. Nine solitary and 5 colonial species are present, 13 of them in San Diego Bay, the largest bay in this region. The most northerly and among the smallest bays, Santa Barbara, contains 8 nonindigenous species. This study reports additional surveys to our 1998 paper (Lambert \& Lambert 1998, Mar Biol 130:675-688) for fall 1997, spring 1998 and summer 2000, documents the newly arrived Botrylloides perspicuum, and corrects the records of 2 species. A main focus is the listing and abundance of species at each survey site within each major harbor and an attempt to analyze the differences between sites and between seasons within a single harbor with reference to variations in water movement, temperature, salinity, pollution factors and competition. Several species appeared for the first time in southern California during 1994 to 1997, apparently correlated with the influx of warmer water during the El Niño of that time. Subsequent surveys show that all of these species have persisted, several have dramatically increased in abundance and distribution, and an additional species has successfully invaded.
\end{abstract}

KEY WORDS: Nonindigenous · Biofouling · Ascidian · Tunicate $\cdot$ Southern California $\cdot$ NE Pacific

\section{INTRODUCTION}

The Southern California Bight (SCB) covers a region of complex currents along approximately $300 \mathrm{~km}$ of the Mexican and southern California shoreline, extending from Cabo Colnett in Baja California northward to Point Conception, California (Seapy 1974, Dailey et al. 1993). The number of humans living near the shore has increased from fewer than 200000 in 1900 to well over 17000000 in 1998; this has resulted in loss of habitat, a tremendous increase in bacteria in nearshore areas and the dredging of numerous harbors and bays (Schiff et al. 2000). These bays contain extensive pilings, breakwaters and floating docks for moorage of thousands of small boats and large cargo ships. All of these artificial surfaces present an immense area of substrate for fouling organisms (Glasby \& Connell 1999). Fouling communities are often dominated by ascidians (Lambert \& Lambert 1998, Lambert 2001, 2003), which are highly efficient filter feeders that thrive on anthropogenically generated bacteria (Bak et al. 1996) and seston (Coma et al. 2001). Most of the native species found in harbors by Ritter \& Forsyth (1917), who carried out the first large-scale survey of ascidians in southern California in 1915, no longer inhabit these areas, though they are still abundant on natural substrates outside the harbors (Fay \& Johnson 1971, Fay \& Vallee 1979); this indicates that large changes have occurred in these harbors. 
The SCB has several large harbors containing one to several marinas (assemblages of floating docks for mooring boats), including one of the world's largest the Long Beach Marina in Alamitos Bay. Our previous study (Lambert \& Lambert 1998) included over $35 \mathrm{yr}$ of observations up to spring 1997 on the occurrence of indigenous and nonindigenous ascidians in these waters. Nearly all the ascidians in southern California harbors are now nonindigenous (Lambert \& Lambert 1998). We will never know which ascidians were present before the Spanish explorer Juan Cabrillo entered San Diego Bay in 1541, but it is likely that some species now considered indigenous arrived from elsewhere centuries ago due to anthropogenic activities.

Semi-numerical data were collected during 1994 to 1997 (Lambert \& Lambert 1998), which included a major El Niño warming of the waters off southern California that may have favored certain species (Stachowicz et al. 2002). In our previous study we combined data from all survey sites within each of the 12 major harbors to give an overall account per harbor of the occurrence and relative abundance of the introduced ascidians. We now report on differences between sites within each harbor and analyze the possible reasons for these differences. New data are presented from surveys carried out during fall 1997, spring 1998 and summer 2000; the latter date includes 2 sites in Ensenada (Baja California), approximately $100 \mathrm{~km}$ south of San Diego. The tables incorporate 2 taxonomic changes from our 1998 paper, based on new knowledge of 2 of the species and a re-examination of preserved material. We correct some of the Botrylloides diegensis records from our 1998 study that included the unrecognized congener $B$. violaceus, change the listings of Symplegma brakenhielmi to additional

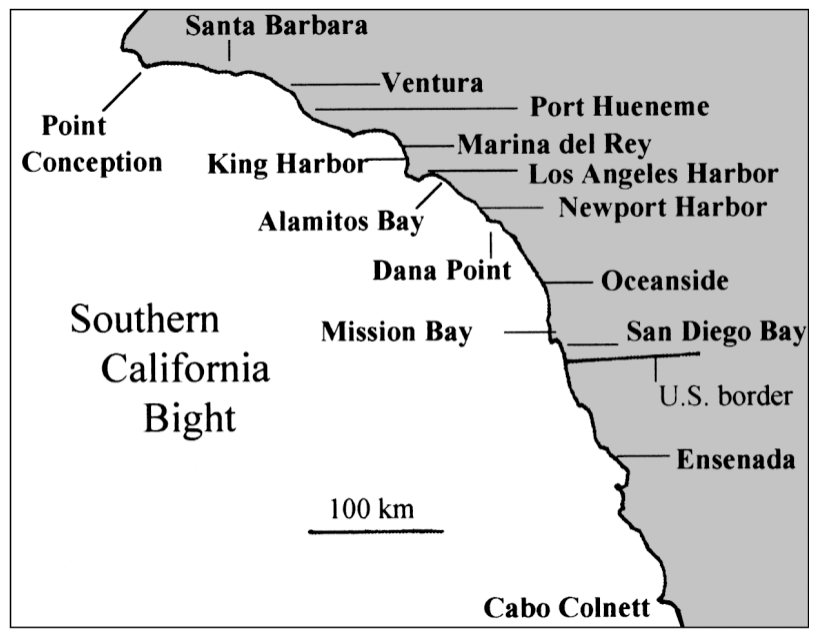

Fig. 1. Southern California Bight with location of study sites records for $S$. reptans, and follow the spread of several other species into additional harbors. The most important findings are the persistence of the 13 nonindigenous species present in 1997 (Lambert \& Lambert 1998) during the subsequent return of cooler waters and the arrival in fall 1997 and spread of an additional species, B. perspicuum, known previously from the tropical western Pacific (Monniot \& Monniot 1996, 2001), Australia (Kott 1985) and Belize (Goodbody 2000). Only 3 of these 14 species were known from southern California in 1945 (Van Name 1945).

\section{MATERIALS AND METHODS}

The methods outlined in Lambert \& Lambert (1998) were used to determine the relative abundance of 14 species of nonindigenous ascidians in the 12 major harbors of southern California, from San Diego to Santa Barbara (Fig. 1). The surveys covered a total of 29 sites within these harbors, with 182 determinations of abundance, carried out in fall 1994, spring and fall 1995, fall 1996, spring and fall 1997, spring 1998 and summer 2000, plus 2 sites in Ensenada, Baja California, sampled once in August 2000. The spring surveys recorded which species overwintered in the marinas or were able to re-form float populations from survivors in deeper regions of the harbors after the winter rains brought an influx of fresh water into most of these harbors. The fall surveys monitored which species had been able to grow through the summer and survive the warmest months of the year. We examined the sides and bottom edges of numerous floats from different areas in the marinas, manually removed aggregated clumps of foulers from float and boat bottoms as far as we could reach, and pulled up our previously installed ropes and any other submerged ropes or wires found. Most of the $5 \mathrm{~m}$ ropes installed in fall 1996 were still in place in August 2000. Each site was searched thoroughly until no additional species were collected, which might take $2 \mathrm{~h}$ at a large marina. We included floats nearest and farthest from shore at every site. Any species not instantly identifiable were taken back to the laboratory at California State University Fullerton and maintained alive in cooled aerated seawater aquaria until they could be examined. The authors maintain a voucher collection of preserved specimens.

Abundance was designated in a semi-quantitative manner: blank - the species was not found after a thorough search (at least $1 \mathrm{~h}$, much longer in larger marinas); 1 - present but rare $\left(<0.1 \mathrm{~m}^{-2}\right) ; 2$ - common $\left(\sim 1 \mathrm{~m}^{-2}\right) ; 3$-abundant $\left(\sim 10 \mathrm{~m}^{-2}\right) ; 4$-complete coverage of large portions of substrate $\left(>100 \mathrm{~m}^{-2}\right)$. All statistical tests of relative abundances were performed with Instat version 2.0 software (Graphpad 1993). 
Gonadal location was used in distinguishing the genus Botryllus from Botrylloides (Saito et al. 2001). The nonindigenous Botrylloides violaceus was not recognized as a separate species co-occurring with the native Botrylloides diegensis in southern California harbors until 1997 because of the highly variable description of $B$. diegensis in Van Name (1945), the reference originally used for identification of this species. However, the 2 species can be distinguished in the field by the colony pigmentation pattern and larval morphology. B. diegensis colonies are always bicolored with a bright white or yellow ring around the oral siphons; $B$. violaceus is always a single color, including various shades of orange, purple, lavender, red or brown. In mature colonies the size and appearance of the incubated larvae are remarkably different in the 2 species, though larvae are not always present and indeed are rarely found in B. diegensis. $B$. violaceus has a large larva ( $1.2 \mathrm{~mm}$ in body diameter) with 24 to 32 ampullae that develops in an incubatory pouch in the tunic (Saito et al. 1981), while B. diegensis larvae are much smaller with only 8 ampullae and are incubated inside the adult zooids. Where we were certain of the identification based on field notations or a reexamination of preserved material, we include abundance data for both species, otherwise a listing as 'nd' is given (see Table 3).

The recently introduced Botrylloides perspicuum is easily distinguished from both $B$. diegensis and $B$. violaceus; the zooids of $B$. perspicuum are much longer, with about 18 rows of stigmata (Monniot \& Monniot 1996, 2001). In the field it can be recognized by its much thicker and firmer tunic and the prominent ridges of tunic that separate groups of zooids. Larvae were not observed.

\section{RESULTS}

\section{Distribution and persistence of introduced species within the Southern California Bight}

All of the introduced species reported in our previous paper (Lambert \& Lambert 1998) except Bostrichobranchus pilularis are still present in the SCB, plus 1 additional species first recorded in fall 1997 (Table 1). Table 2 lists the 14 nonindigenous species and percent of the 29 sites in 13 harbors in which they were observed during this study. Nine (64\%) of the introduced ascidians are solitary, $5(36 \%)$ are colonial. Ten (71\%) are stolidobranchs, 4 (29\%) are phlebobranchs; surprisingly, there are none from the order Aplousobranchia. Styela plicata, the most abundant invader, present at $100 \%$ of our study sites (Tables $2 \& 3$ ), is also one of the first to have been recognized as nonindigenous, by Ritter \& Forsyth (1917). The dominant biomass of fouling organisms is $S$. plicata at most of these sites. Styela clava, present for at least $70 \mathrm{yr}$, is another species present and often very abundant at all sites. Although there is a trend for the species present the longest to have the widest distribution, there are some notable exceptions. Ciona savignyi, Microcosmus squamiger, and Ascidia zara have been known in this region for less than $20 \mathrm{yr}$, yet have a wide distribution, while Styela canopus even after 30 or more years is still confined to a few sites (Table 2). Ascidia sp. decreased in abundance after fall 1997 north of San Diego (Table 3). Nevertheless, the overwhelming trend is for long-term persistence of introduced species; indeed 2 of the species, C. intestinalis and S. plicata, have now been present since 1915 (Table 2). The

Table 1. Nonindigenous ascidians in Southern California Bight harbors recorded at least once between 1994 and 2000 (listed from south to north). For complete data showing major differences among the 29 sites, and dates of the most recently introduced species, see Table 3. C. in = Ciona intestinalis, C. sa = Ciona savignyi, A. za = Ascidia zara, A. sp. = Ascidia sp., S. ca= Styela canopus (formerly S. partita), S. cl=Styela clava, S. pl=Styela plicata, P. zo = Polyandrocarpa zorritensis, B. sC $=$ Botryllus schlosseri, B. per = Botrylloides perspicuum, B. vi = Botrylloides violaceus, $S$. re = Symplegma reptans, M. sq = Microcosmus squamiger, M. ma = Molgula manhattensis

\begin{tabular}{|c|c|c|c|c|c|c|c|c|c|c|c|c|c|c|}
\hline Location & C. in & C. sa & A. za & A. sp. & S. ca & S. $C l$ & S. $p l$ & P. zo & B. $s C$ & B. per & B. vi & S. re & M. $s q$ & M. ma \\
\hline Ensenada, Baja California & + & & & & & + & + & + & + & & + & & + & \\
\hline San Diego Bay & + & + & + & + & + & + & + & + & + & + & + & + & + & \\
\hline Mission Bay & + & + & + & + & + & + & + & + & + & + & + & + & + & \\
\hline Oceanside Harbor & & + & + & & + & + & + & + & + & & + & & + & \\
\hline Dana Point & + & & + & & & + & + & & + & & + & + & + & \\
\hline Newport Harbor & + & + & + & + & + & + & + & & + & & + & & + & + \\
\hline Alamitos Bay & + & + & + & + & + & + & + & + & + & & + & & + & + \\
\hline Long Beach/Los Angeles Harbors & $s+$ & + & + & + & + & + & + & + & + & & + & + & + & + \\
\hline King Harbor & + & + & + & + & & + & + & + & + & & + & & + & \\
\hline Marina Del Rey & + & + & + & + & & + & + & & + & & + & & + & + \\
\hline Port Hueneme & + & + & + & + & & + & + & & + & & + & & + & \\
\hline Ventura Harbor & + & + & + & + & & + & + & & + & & + & & + & + \\
\hline Santa Barbara Harbor & + & + & + & + & & + & + & & + & & + & & & \\
\hline
\end{tabular}


Table 2. Percent of 29 study sites occupied by nonindigenous ascidians in southern California harbors and year of first report. Order: $\mathrm{P}=$ Phlebobranchia, $\mathrm{S}=$ Stolidobranchia

\begin{tabular}{|c|c|c|c|c|c|}
\hline Species in rank order & $\%$ sites & Year of first report & Order & Colonial/Solitary & Source \\
\hline Styela clava & 100 & 1933 & $\mathrm{~S}$ & $\mathrm{~s}$ & Abbott \& Johnson (1972) \\
\hline Styela plicata & 100 & 1915 & $\mathrm{~S}$ & $\mathrm{~s}$ & Ritter \& Forsyth (1917) \\
\hline Botryllus schosseri & 100 & $1965 ?$ & $\mathrm{~S}$ & $\mathrm{C}$ & Lambert \& Lambert (1998) \\
\hline Ciona savignyi & 96.5 & 1985 & $\mathrm{P}$ & $\mathrm{s}$ & Lambert \& Lambert (1998) \\
\hline Microcosmus squamiger & 96.5 & 1986 & $\mathrm{~S}$ & $\mathrm{~s}$ & Lambert \& Lambert (1998) \\
\hline Botrylloides violaceus & 90 & $1945^{\mathrm{a}}$ & $\mathrm{S}$ & $\mathrm{C}$ & Lambert \& Lambert (1998) \\
\hline Ciona intestinalis & 86.2 & 1915 & $\mathrm{P}$ & $\mathrm{s}$ & Ritter \& Forsyth (1917) \\
\hline Ascidia zara & 75.9 & 1984 & $\mathrm{P}$ & $\mathrm{s}$ & Lambert \& Lambert (1998) \\
\hline Ascidia sp. & 62.1 & 1983 & $\mathrm{P}$ & $\mathrm{s}$ & Lambert \& Lambert (1998) \\
\hline Styela canopus & 48.3 & 1972 & $\mathrm{~S}$ & $\mathrm{~s}$ & Lambert \& Lambert (1998) \\
\hline Polyandrocarpa zorritensis & 44.8 & 1994 & $\mathrm{~S}$ & $\mathrm{C}$ & Lambert \& Lambert (1998) \\
\hline Symplegma reptans & 37.9 & 1991 & $\mathrm{~S}$ & $\mathrm{C}$ & Lambert \& Lambert (1998) \\
\hline Molgula manhattensis & 37.9 & 1984 & $\mathrm{~S}$ & $\mathrm{~s}$ & Lambert \& Lambert (1998) \\
\hline Botrylloides perspicuum & 10.3 & 1997 & $\mathrm{~S}$ & $\mathrm{C}$ & Present study \\
\hline
\end{tabular}

abundance of Botryllus schlosseri is probably greater than recorded in Table 3. Even though the species is present at all monitoring sites, the bright orange colonies were probably recorded more accurately than the small black highly cryptic colonies.

The species designated Symplegma brakenhielmi in our 1998 paper based on fragments of small immature colonies collected in 1991 and 1994 from Los Angeles and San Diego Bays is now recognized as the first east Pacific records for the Japanese species Symplegma reptans. It underwent a huge population explosion in 1997 and 1998 in San Diego and Mission Bays, and spread north to Dana Point and Long Beach, its northernmost California record so far (Table 3).

Polyandrocarpa zorritensis was already present at Oceanside and throughout San Diego and Mission Bays in 1994. Subsequently it has spread north to a few Los Angeles harbors. The newest invader, Botrylloides perspicuum, was first recorded from San Diego Bay in fall 1997; by August 2000 it had spread to a second site in the bay and also to Mission Bay, and its biomass had increased dramatically.

Although there were wide variations from year to year both within and between harbors, Ascidia sp. is more abundant in the fall, as shown by the MannWhitney $U$-test. Within San Diego Bay, Ciona intestinalis was also statistically more abundant in the spring than the fall, with a mean of $2.26 \pm 0.244$ compared to the fall mean of $0.812 \pm 0.122$. Polyandrocarpa zorritensis shows a trend toward higher abundance in the fall; certainly the individual colonies were much larger in the fall. Ciona savignyi and Ascidia zara reverse this trend with somewhat greater abundance in the spring.
The same 13 nonindigenous species occur in both San Diego and Mission Bays; there are 8 to 12 species in the SCB harbors north of San Diego and 7 in Ensenada (Table 1). There appear to be definite northsouth species distributions for several of the ascidians (Table 3). The most recent invader, Botrylloides perspicuum, has not yet appeared in any harbors north of San Diego nor was it found in Ensenada in August 2000. Symplegma reptans and Styela canopus are also apparently absent from Ensenada but common in San Diego, present in a few harbors north of San Diego County but absent from all harbors north of Los Angeles; S. Canopus is, in fact, rare anywhere north of San Diego. Polyandrocarpa zorritensis is also absent from all harbors north of Los Angeles but was abundant in Ensenada in August 2000. Ciona intestinalis, C. savignyi, Styela clava, S. plicata and Microcosmus squamiger are present in most SCB harbors, and all but $C$. savignyi were recorded from Ensenada in August 2000. M. squamiger is now rare north of Los Angeles and has never been recorded from Santa Barbara. At the present time Molgula manhattensis is not known to occur south of Newport Bay, and where it does occur is restricted to quiet waters at the backs of harbors.

\section{Major highlights of the individual harbors (Fig. 1, Table 3)}

\author{
Ensenada, Baja California
}

We sampled 2 small marinas, Juanita's Dock at the Sportfishing Marina and the Hotel Coral Marina. 
Table 3. Occurrence and abundance of introduced ascidians from San Diego to Santa Barbara, 1994-2000 and Ensenada (Baja California), August 2000. The monitoring site closest to the entrance in each major bay is listed first, and the innermost site is listed last. $C$. in = Ciona intestinalis, C. sa = Ciona savignyi, A. za = Ascidia zara, A. sp. = Ascidia sp., S. ca = Styela canopus (formerly S. partita) S. $\mathrm{cl}=$ Styela clava, S. pl = Styela plicata, $P$. zo = Polyandrocarpa zorritensis, B. sC = Botryllus schlosseri, B. $p e=$ Botrylloides perspicuum, B. vi = Botrylloides violaceus, $S$. re = Symplegma reptans, M. sq = Microcosmus squamiger, M. ma $=$ Molgula manhattensis. 1: present but rare $\left(<0.1 \mathrm{~m}^{-2}\right) ; 2$ : common $\left(\sim 1 \mathrm{~m}^{-2}\right)$; 3: abundant $\left(\sim 10 \mathrm{~m}^{-2}\right) ; 4:$ complete cover of large portions of substrate $\left(>100 \mathrm{~m}^{-2}\right)$, nd: not determined

\begin{tabular}{|c|c|c|c|c|c|c|c|c|c|c|c|c|c|}
\hline Location, time & C. in & C. sa & A. za & A. sp. & S. ca & S. $C l$ & S. $p l$ & P. zo & B. $S C$ & B. pe & B. vi & S. re & M. sq M. ma \\
\hline \multicolumn{14}{|c|}{ Ensenada, Baja California, August 2000} \\
\hline Juanita's Dock & 3 & & & & & 4 & 4 & 3 & 3 & & 3 & & \\
\hline Hotel Coral & 1 & & & & & 4 & 4 & 3 & 1 & & & & 1 \\
\hline \multicolumn{14}{|l|}{ San Diego Bay } \\
\hline \multicolumn{14}{|l|}{ Shelter Island } \\
\hline Fall 1994 & & & & 1 & & 1 & 4 & 4 & 1 & & nd & & 1 \\
\hline Spring 1995 & 2 & 2 & 1 & & 2 & & 2 & 3 & nd & & 1 & & \\
\hline Fall 1995 & 1 & & & & 1 & 3 & 2 & 3 & 2 & & nd & & 1 \\
\hline Fall 1996 & 1 & & & 1 & & & 4 & 2 & 1 & & & & 1 \\
\hline Spring 1997 & 3 & & 1 & & & 2 & 3 & 3 & 1 & & 1 & & 2 \\
\hline Fall 1997 & 2 & & 1 & 2 & & 2 & 4 & 4 & 1 & & nd & & \\
\hline Spring 1998 & 2 & & 1 & & & 3 & 4 & 3 & 2 & 1 & 1 & & 3 \\
\hline August 2000 & 3 & 3 & 1 & 2 & 2 & 3 & 4 & 4 & 3 & 2 & 2 & 2 & \\
\hline \multicolumn{14}{|l|}{ Harbor Island } \\
\hline Fall 1994 & 1 & 1 & & 3 & & 1 & 1 & 1 & & & & & 1 \\
\hline Spring 1995 & 2 & 2 & 3 & 1 & & 2 & 3 & 2 & 2 & & 2 & & 2 \\
\hline Fall 1996 & & 2 & 2 & 3 & & & 1 & 4 & 1 & & nd & & 1 \\
\hline Spring 1997 & 3 & 2 & 3 & & & 2 & 3 & 2 & 1 & & nd & & 1 \\
\hline Fall 1997 & 1 & 1 & & 3 & & 1 & 3 & 4 & 1 & & nd & & \\
\hline Spring 1998 & 3 & 4 & 4 & 1 & 1 & & 3 & 4 & & & 2 & & 1 \\
\hline \multicolumn{14}{|l|}{ Fiddlers Cove } \\
\hline Fall 1994 & & 2 & & & 2 & & 3 & 3 & nd & & nd & & \\
\hline Spring 1995 & 2 & 2 & & & 3 & 3 & 3 & 2 & 2 & & 3 & 1 & 3 \\
\hline Fall 1995 & & 3 & 1 & & 3 & 3 & 3 & 3 & 1 & & nd & & 3 \\
\hline Fall 1996 & & & 1 & 1 & 2 & 1 & 1 & 1 & 1 & & 1 & & 4 \\
\hline Spring 1997 & 2 & 1 & & & 3 & & 1 & 1 & 1 & & 1 & & 4 \\
\hline Fall 1997 & 1 & 1 & & 2 & 3 & 1 & 1 & 3 & 1 & & nd & 1 & 4 \\
\hline Spring 1998 & 3 & 1 & 1 & & 3 & 3 & 3 & 3 & 3 & & 2 & 4 & 4 \\
\hline August 2000 & 2 & 3 & & 1 & 4 & 4 & 3 & 4 & 1 & & 1 & 1 & 4 \\
\hline \multicolumn{14}{|l|}{ 24th Street } \\
\hline Fall 1994 & & 2 & & 1 & 2 & & 2 & 2 & 1 & & nd & 2 & 4 \\
\hline \multicolumn{14}{|c|}{ Spring 1995 (dredged) } \\
\hline Fall 1995 & 1 & 1 & & & 1 & & & 3 & & & & & 1 \\
\hline Fall 1996 & 1 & & & & 2 & 1 & 1 & & & & & & 4 \\
\hline Spring 1997 & 3 & 3 & & & & 2 & 2 & & & & nd & & \\
\hline Fall 1997 & 3 & & & 1 & 2 & 1 & 1 & 3 & & 4 & nd & & 4 \\
\hline Spring 1998 & 1 & & & & & & 1 & 3 & 1 & 3 & nd & & 3 \\
\hline \multicolumn{14}{|l|}{ J Street } \\
\hline Fall 1994 & & & & 3 & 2 & & 3 & 2 & & & 1 & & 2 \\
\hline Spring 1995 & 2 & 3 & nd & & 2 & 2 & 1 & & 1 & & nd & & 3 \\
\hline Fall 1995 & 1 & & nd & 1 & & & 1 & 3 & & & 1 & & 3 \\
\hline Fall 1996 & & & & 1 & 2 & & 1 & & & & 1 & & 3 \\
\hline Spring 1997 & 3 & 1 & 1 & & 1 & 1 & 1 & 1 & 1 & & 1 & & 4 \\
\hline Fall 1997 & & 1 & & 3 & 3 & & 2 & 2 & 2 & & nd & & 2 \\
\hline Spring 1998 & 4 & 1 & & 1 & 2 & 1 & 2 & 4 & 2 & & 2 & & 4 \\
\hline August 2000 & 1 & 4 & & 3 & 4 & 1 & 4 & 3 & & & 1 & & 4 \\
\hline \multicolumn{14}{|l|}{ Mission Bay } \\
\hline \multicolumn{14}{|l|}{ Quivera Basin } \\
\hline Fall 1994 & 2 & & & 2 & & 1 & 2 & & 1 & & 1 & & \\
\hline Spring 1995 & 4 & 2 & 1 & & & 1 & 2 & 1 & nd & & 1 & & \\
\hline Fall 1995 & 1 & 1 & & 1 & & 1 & 1 & & & & nd & & 1 \\
\hline Fall 1996 & 2 & & 1 & 1 & & 1 & 4 & 3 & 1 & & nd & & 1 \\
\hline Spring 1997 & 3 & 1 & 1 & & & 1 & 3 & 2 & 1 & & nd & & \\
\hline Fall 1997 & 1 & & 1 & 2 & & 3 & 4 & 3 & & & & 1 & 1 \\
\hline
\end{tabular}


Table 3 (continued)

\begin{tabular}{|c|c|c|c|c|c|c|c|c|c|c|c|c|c|c|}
\hline Location, time & C. in & C. sa & A. za & A. sp. & S. ca & S. $\mathrm{Cl}$ & S. $p l$ & P. zo & B. $S C$ & B. pe & B. vi & S. re & M. sq & M. ma \\
\hline Spring 1998 & 4 & 3 & 2 & 1 & & 3 & 4 & 1 & 2 & 1 & nd & 2 & 1 & \\
\hline August 2000 & 4 & 2 & 1 & 3 & & 3 & 4 & 2 & 2 & 3 & 1 & & 1 & \\
\hline \multicolumn{15}{|l|}{ Dana Landing } \\
\hline Fall 1994 & & 1 & & & & & 2 & 2 & 1 & & nd & & 3 & \\
\hline Spring 1995 & & 2 & & & & 3 & 2 & & & & 1 & & 3 & \\
\hline Fall 1995 & & & & & & 1 & 1 & & 1 & & 1 & & 1 & \\
\hline Fall 1996 & & 1 & & 1 & 1 & 1 & 1 & & 1 & & nd & & 2 & \\
\hline Spring 1997 & & & & & 2 & 3 & 3 & & 1 & & nd & & 2 & \\
\hline Fall 1997 & & & & & & 1 & 2 & 1 & 2 & & nd & & 3 & \\
\hline Spring 1998 & 1 & & & & 2 & 3 & 3 & & 3 & & nd & 1 & 4 & \\
\hline \multicolumn{15}{|c|}{ South Shores Boat Ramp } \\
\hline Spring 1997 & 3 & 1 & & & 3 & 2 & 3 & & 1 & & nd & 3 & 3 & \\
\hline Fall 1997 & 1 & & & & & 1 & 2 & 4 & 3 & & & 2 & 3 & \\
\hline Spring 1998 & & 2 & & & & 1 & 3 & 3 & 2 & & 4 & & 2 & \\
\hline \multicolumn{15}{|l|}{ Bahia Point } \\
\hline Fall 1994 & & & & 1 & & 2 & 2 & 3 & 1 & & nd & & 3 & \\
\hline Spring 1995 & & & & & & 3 & 2 & & nd & & nd & & 3 & \\
\hline Fall 1995 & & & & & & 1 & 3 & 1 & & & nd & & 2 & \\
\hline Fall 1996 & 1 & 1 & 1 & & & 1 & 2 & 3 & & & nd & & 2 & \\
\hline Spring 1997 & 2 & & & 1 & 1 & 4 & 4 & & & & nd & 2 & 4 & \\
\hline Fall 1997 & & & & & & 2 & & 4 & & & nd & 1 & 3 & \\
\hline Spring 1998 & 1 & 2 & 2 & & & 3 & 4 & 1 & 2 & & 2 & 2 & 1 & \\
\hline \multicolumn{15}{|c|}{ Mission Bay Yacht Club } \\
\hline Spring 1997 & 1 & 1 & 1 & & & 3 & & & 1 & & 1 & 3 & 2 & \\
\hline Fall 1997 & & & 1 & & & 2 & 3 & & 1 & & nd & 3 & 3 & \\
\hline Spring 1998 & 1 & 2 & 1 & & & 3 & 2 & & 2 & & nd & 4 & 2 & \\
\hline \multicolumn{15}{|c|}{ Santa Clara Boat Launch } \\
\hline Spring 1997 & & 1 & 1 & & & 2 & 1 & & 1 & & nd & & 2 & \\
\hline Fall 1997 & & & & & & 1 & 1 & & & & nd & 1 & 3 & \\
\hline Spring 1998 & & 2 & 2 & & & 2 & 2 & & 1 & & nd & 2 & 1 & \\
\hline \multicolumn{15}{|l|}{ Oceanside } \\
\hline \multicolumn{15}{|l|}{ Police Floats } \\
\hline Fall 1994 & & 1 & & & & & & 2 & & & & & 2 & \\
\hline Spring 1995 & & & nd & & & 1 & 3 & & & & & & 2 & \\
\hline Fall 1995 & & & & & & 1 & 1 & 1 & 1 & & & & 2 & \\
\hline Fall 1996 & & 1 & & & 1 & 2 & 2 & 2 & 2 & & 1 & & 2 & \\
\hline Spring 1997 & & & & & & 1 & 2 & 1 & & & & & 2 & \\
\hline Fall 1997 & & & & & & & 3 & 1 & 2 & & & & 3 & \\
\hline Spring 1998 & & & 1 & & & 2 & 2 & & & & & & 3 & \\
\hline August 2000 & & & & & & 2 & 3 & 4 & 2 & & & & 2 & \\
\hline \multicolumn{15}{|l|}{ Back Harbor } \\
\hline Fall 1994 & & 2 & & & & & & & & & & & & \\
\hline Fall 1995 & & & & & & 1 & & & & & & & & \\
\hline Spring 1997 & & & & & & 1 & 3 & & & & & & 1 & \\
\hline Fall 1997 & & & & & 1 & & 4 & & 2 & & 1 & & & \\
\hline Spring 1998 & & 1 & & & & & 3 & & 1 & & & & & \\
\hline \multicolumn{15}{|l|}{ Dana Point } \\
\hline Fall 1994 & & & & & & 1 & 2 & & 2 & & & & 2 & \\
\hline Fall 1996 & 1 & & 1 & & & 1 & 1 & & 1 & & & & 1 & \\
\hline Spring 1997 & & & & & & & 2 & & & & & & 2 & \\
\hline Fall 1997 & & & & & & & 3 & & 1 & & & 2 & 2 & \\
\hline Spring 1998 & & & & & & & 3 & & 2 & & & 1 & 2 & \\
\hline \multicolumn{15}{|l|}{ Newport Harbor } \\
\hline \multicolumn{15}{|l|}{ Fun Zone } \\
\hline Fall 1994 & & & & & & 2 & 2 & & & & & & 2 & \\
\hline Fall 1995 & 3 & & & & & & 3 & & & & & & & \\
\hline Fall 1996 & & & & & & 2 & 2 & & 1 & & & & 1 & \\
\hline Spring 1997 & & 1 & 1 & & 1 & 3 & 1 & & & & 1 & & 2 & 2 \\
\hline Fall 1997 & & & & & & 1 & 1 & & & & & & 3 & 1 \\
\hline Spring 1998 (rain) & & & & & & & & & & & & & & \\
\hline
\end{tabular}


Table 3 (continued)

\begin{tabular}{|c|c|c|c|c|c|c|c|c|c|c|c|c|c|c|}
\hline Location, time & C. in & C. sa & A. za & A. sp. & S. Ca & S. $\mathrm{Cl}$ & S. $p l$ & P. zo & B. $S C$ & B. pe & B. vi & S. re & M. $s q$ & M. ma \\
\hline \multicolumn{15}{|l|}{ Lido Island } \\
\hline \multicolumn{15}{|l|}{ Fall 1994 (oil spill) } \\
\hline Fall 1995 & & & & & & 1 & & & & & & & 1 & 2 \\
\hline Fall 1996 & 4 & 1 & & & & & & & & & & & & \\
\hline Spring 1997 & 4 & 4 & & & & 2 & & & & & & & & 2 \\
\hline Fall 1997 & 3 & & & & & & 1 & & 1 & & & & & \\
\hline Spring 1998 (rain) & 1 & 1 & & & & & & & 1 & & & & & 4 \\
\hline \multicolumn{15}{|l|}{ Upper Bay } \\
\hline Fall 1994 & 1 & 1 & & & & 3 & 3 & & & & & & & 3 \\
\hline Fall 1995 & 4 & & & & & & 2 & & & & & & & 1 \\
\hline Fall 1996 & & 1 & 1 & & & 3 & 3 & & 1 & & & & 3 & 2 \\
\hline Spring 1997 (dreds & & & & & & 1 & & & & & & & & 1 \\
\hline $\begin{array}{l}\text { Fall } 1997 \\
\text { Spring } 1998 \text { (rain) }\end{array}$ & 2 & 2 & 1 & & & 3 & 3 & & 1 & & & & 2 & 2 \\
\hline \multicolumn{15}{|l|}{ Long Beach Marina } \\
\hline \multicolumn{15}{|l|}{ Entrance } \\
\hline Fall 1994 & & & & & & 1 & & & & & nd & & 2 & \\
\hline Fall 1995 & 1 & & nd & 1 & & & & & 1 & & nd & & 1 & \\
\hline Fall 1996 & 1 & 1 & & & & & & & & & nd & & & 1 \\
\hline Spring 1997 & 1 & & nd & & & & & & 1 & & nd & & & \\
\hline Fall 1997 & 1 & & & & & 1 & 1 & & 1 & & nd & & 1 & \\
\hline Spring 1998 (rain) & & & & & & & & & & & 1 & & 1 & \\
\hline \multicolumn{15}{|l|}{ Back Harbor } \\
\hline Fall 1994 & & & & & & 2 & 2 & & 2 & & nd & & 2 & \\
\hline Fall 1995 & & & & & & 1 & 1 & & 1 & & nd & & 3 & \\
\hline Fall 1996 & & 1 & & & & 2 & 2 & & 2 & & nd & & 2 & \\
\hline Spring 1997 & & 1 & & 2 & & 3 & & & 2 & & nd & & 1 & 1 \\
\hline Fall 1997 & & 2 & & & & 3 & 1 & 3 & 3 & & nd & & 3 & \\
\hline Spring 1998 & & & & & & 3 & 3 & & 3 & & 3 & & 3 & 1 \\
\hline August 2000 & 2 & 1 & & 1 & 1 & 3 & 1 & 1 & 3 & & 3 & & 1 & \\
\hline \multicolumn{15}{|l|}{ Spinnaker Cove } \\
\hline Fall 1997 & & 3 & 3 & & 2 & 3 & 4 & & 1 & & nd & & 3 & \\
\hline Spring 1998 & & & & & & 3 & 3 & & & & 1 & & & 4 \\
\hline \multicolumn{15}{|c|}{ Long Beach/Los Angeles Harbors } \\
\hline \multicolumn{15}{|c|}{ Impound Marina } \\
\hline Fall 1994 & 3 & 2 & nd & & & 3 & 2 & & 2 & & nd & & 1 & \\
\hline Spring 1995 & 1 & 1 & 2 & & & 1 & 1 & & 1 & & 1 & & 1 & \\
\hline Fall 1995 & 3 & 1 & & & & 2 & 2 & & 2 & & nd & & & \\
\hline Fall 1996 & 2 & 1 & 2 & & & 1 & 2 & & 2 & & 1 & & 2 & \\
\hline Spring 1997 & 1 & 2 & 2 & & & 1 & 3 & & 2 & & nd & 1 & 2 & \\
\hline Fall 1997 & 3 & 3 & 3 & 1 & & 3 & 3 & & 3 & & nd & 2 & 1 & \\
\hline Spring 1998 & 4 & 4 & 3 & & & 3 & 3 & 3 & 2 & & nd & 2 & 1 & 1 \\
\hline August 2000 & 4 & & & & & 4 & 4 & 2 & 2 & & 3 & & & \\
\hline \multicolumn{15}{|l|}{ Watchorn Marina } \\
\hline Fall 1994 & 1 & 1 & & & & & 1 & & 1 & & 1 & & & \\
\hline Spring 1995 & 1 & 1 & 1 & & & & 1 & & 1 & & nd & & & \\
\hline Fall 1996 & 2 & 1 & 1 & & & 1 & 1 & & 1 & & nd & & & \\
\hline Spring 1997 & 2 & 3 & 3 & & & 1 & 1 & & 1 & & nd & & & \\
\hline Fall 1997 & 3 & 2 & 3 & & & 2 & 2 & & $\begin{array}{l}1 \\
3\end{array}$ & & nd & & & 1 \\
\hline Spring 1998 & 4 & 4 & 4 & & 1 & 2 & 3 & & 3 & & nd & & & 1 \\
\hline August 2000 & 4 & 1 & 2 & & & 4 & 4 & & 3 & & 4 & & 1 & \\
\hline \multicolumn{15}{|l|}{ King Harbor } \\
\hline Fall 1994 & 3 & & nd & 1 & & 2 & 2 & & 1 & & nd & & 1 & \\
\hline \multicolumn{15}{|l|}{ Spring 1995} \\
\hline Fall 1995 & 2 & & & & & 1 & 2 & & 2 & & nd & & 1 & \\
\hline Fall 1996 & 2 & 1 & & 1 & & & 2 & & 1 & & nd & & & \\
\hline Spring 1997 & 2 & 1 & & & & 1 & 3 & & 2 & & nd & & 1 & \\
\hline Fall 1997 & 3 & 1 & & 4 & & 1 & 4 & 1 & 4 & & nd & & 2 & \\
\hline Spring 1998 & 3 & 1 & 1 & & & & 3 & 1 & 3 & & & & 2 & \\
\hline August 2000 & 2 & & & & & 1 & 4 & 3 & 2 & & 1 & & 1 & \\
\hline
\end{tabular}


Table 3 (continued)

\begin{tabular}{|c|c|c|c|c|c|c|c|c|c|c|c|}
\hline Location, time & C. in & C. sa & A. za & A. sp. S. ca & S. $\mathrm{Cl}$ & S. pl P. zo & B. sC B.pe & B. vi & S. re & M. $s q$ & M. ma \\
\hline \multicolumn{12}{|l|}{ Marina Del Rey } \\
\hline Fall 1994 & 1 & & & 2 & & 3 & $1 ?$ & nd & & & \\
\hline Spring 1995 & 1 & & 1 & & & 4 & 1 & nd & & & 1 \\
\hline Fall 1995 & 1 & 1 & & & & 4 & 1 & nd & & & \\
\hline Fall 1996 & 1 & 1 & 2 & 2 & & 4 & 1 & nd & & & 1 \\
\hline Spring 1997 & 1 & 1 & 2 & & & 4 & & nd & & & 2 \\
\hline Fall 1997 & 2 & 2 & 2 & 2 & & 4 & 3 & nd & & & \\
\hline Spring 1998 & 4 & 3 & 2 & & & 3 & 3 & 1 & & & 1 \\
\hline August 2000 & 3 & & 1 & & 1 & 4 & 1 & 1 & & 1 & \\
\hline \multicolumn{12}{|l|}{ Port Hueneme } \\
\hline \multicolumn{12}{|l|}{ Entrance: Jack's Landing } \\
\hline Fall 1994 & & & & & 2 & & & & & 3 & \\
\hline Fall 1995 & 1 & 1 & nd & 2 & 3 & 1 & 1 & nd & & 1 & \\
\hline Fall 1996 & & 1 & 1 & & 2 & 2 & 2 & nd & & 1 & \\
\hline Spring 1997 & & 1 & & & 4 & & 1 & 2 & & 1 & \\
\hline Fall 1997 (cleaned + replaced) & & & & & & & 2 & nd & & & \\
\hline Spring 1998 & & 1 & 1 & & 1 & & & & & & \\
\hline August 2000 & 3 & & & & 1 & 1 & 1 & & & & \\
\hline Anacapa Island Marina & & & & & & & 4 & 2 & & & \\
\hline Fall 1994 & 1 & 1 & & & 2 & 2 & & & & 3 & \\
\hline Fall 1995 & 1 & 1 & 2 & 2 & 2 & 1 & & nd & & 1 & \\
\hline Fall 1996 & & & 1 & & 1 & 1 & 2 & nd & & 1 & \\
\hline Spring 1997 & & 1 & & 1 & 2 & & & 2 & & & \\
\hline Fall 1997 & 2 & 2 & 2 & & 2 & 3 & & nd & & 1 & \\
\hline Spring 1998 & & 1 & 1 & & & & & nd & & 1 & \\
\hline August 2000 & 3 & 1 & 2 & & 4 & 4 & 1 & 3 & & 1 & \\
\hline Ventura & & & & & & & 1 & 3 & & & \\
\hline \multicolumn{12}{|l|}{ Entrance: Island Packers } \\
\hline Fall 1994 & 1 & & & & 2 & 2 & & & & & \\
\hline Fall 1995 & 3 & & & & 3 & & 2 & nd & & & \\
\hline Fall 1996 & 2 & 2 & 1 & 1 & 4 & 4 & 2 & nd & & 1 & \\
\hline Spring 1997 & 1 & 1 & 1 & & 2 & 2 & 1 & 1 & & 1 & \\
\hline Fall 1997 & 1 & 1 & 1 & & 3 & 3 & 1 & nd & & & \\
\hline Spring 1998 & & 1 & 1 & & 1 & & 1 & nd & & 1 & 1 \\
\hline \multicolumn{12}{|l|}{ Back harbor: Pierpont Yacht Club } \\
\hline Fall 1994 & 1 & & & & 2 & 2 & & & & & 1 \\
\hline Fall 1995 & 1 & & & 1 & 3 & 3 & 2 & nd & & & 2 \\
\hline Fall 1996 & 2 & 2 & 1 & 1 & 4 & 4 & 1 & nd & & & 2 \\
\hline Spring 1997 & 2 & 2 & 3 & & 4 & 4 & 1 & nd & & & 3 \\
\hline Fall 1997 & 2 & 2 & 1 & 1 & 3 & 3 & 1 & nd & & 1 & 3 \\
\hline Spring 1998 & & 3 & 4 & & 2 & 1 & 1 & nd & & 1 & \\
\hline Santa Barbara & & & & & & & 1 & 1 & & & \\
\hline Fall 1994 & 2 & 2 & & & 2 & 4 & & & & & \\
\hline Fall 1995 & 1 & 1 & & 1 & 3 & 3 & & nd & & & \\
\hline Fall 1996 & 1 & 1 & 1 & & 2 & 2 & 1 & nd & & & \\
\hline Spring 1997 & & 2 & & & 2 & 2 & & 2 & & & \\
\hline Fall 1997 & 4 & 1 & & & 4 & 4 & 1 & nd & & & \\
\hline Spring 1998 & 1 & 2 & 2 & & 3 & 3 & 1 & nd & & & \\
\hline
\end{tabular}

Although Ensenada is only about $100 \mathrm{~km}$ south of San Diego, the ascidian diversity is much lower, possibly due to the small size of the marinas (area not measured) and perhaps also to the very polluted appearance of the water at both marinas. Nevertheless, several species were very abundant (Ciona intestinalis, Styela clava, S. plicata, Polyandrocarpa zorritensis, Botryllus schlosseri and Botrylloides vio- laceus), especially below $1 \mathrm{~m}$ on pilings and submerged ropes.

\section{San Diego Bay}

San Diego Bay, the largest bay in the SCB, covers 4441 ha, opening to the sea at its northwest and 
extending southward $15 \mathrm{~km}$ nearly to Mexico. Currents are highest near the entrance with much less water movement in the shallow southern end. The Otay and Sweetwater Rivers empty into the south end of the bay. We monitored 5 sites (Table 3) within the bay from the entrance (Shelter Island) to the south end ( $\mathrm{J}$ Street). This bay has been a major shipping port for several centuries and is also an important naval station and center for thousands of privately owned pleasure boats. In the fall of 1997 large colonies of the western Pacific Botrylloides perspicuum appeared for the first time in the boat launch facility at 24th Street in National City in south San Diego Bay. These colonies occupied several hundred $\mathrm{cm}^{2}$ of the dock sides and bottom. The following spring it was present at Shelter Island and also in Mission Bay. Symplegma reptans, resident since our fall 1994 survey in the south bay, was recorded for the first time at Shelter Island in fall 2000, indicating that it is now present throughout the bay. Microcosmus squamiger and Styela canopus are most abundant in the southern part of the bay. Species consistently more abundant closer to the bay entrance are Ascidia zara and Styela clava, with S. plicata and Polyandrocarpa zorritensis showing a slightly similar tendency though common throughout the bay. Molgula manhattensis does not occur in this bay system but the larger native Molgula verrucifera is always present in several areas.

\section{Mission Bay}

Mission Bay (819 ha) is a shallow, artificially created bay $6 \mathrm{~km}$ north of San Diego Bay; it is the site of former wetlands dredged to provide recreational boating facilities. The long narrow ocean entrance to Mission Bay, separated from the San Diego River by a rock jetty, opens to several major basins connected by channels. No rivers or major streams empty directly into the bay, but freshwater urban runoff as well as seasonal heavy rains occasionally lower the salinity. Because this bay is shallow, both temperature and salinity are much higher in summer than in winter. Of the 6 monitoring sites, 3 (South Shores boat ramp, Mission Bay Yacht Club and Santa Clara boat launch) were added in the spring of 1997, when a large population of Symplegma reptans appeared at the Bahia Point monitoring site. By 1998 it was widespread within the bay, covering mussels and other fouling organisms especially at the Mission Bay Yacht Club. Botrylloides perspicuum was first recorded at Quivera Basin near the entrance to the bay system during the spring 1998 survey; by fall 2000 its abundance had increased several-fold, though it had not yet spread to any additional sites. Styela clava and $S$. plicata have been abundant throughout the bay for many years.

\section{Oceanside Harbor}

Large seasonal changes in ascidian population density occur in Oceanside Harbor (85 ha), located about $21 \mathrm{~km}$ north of Mission Bay, possibly due to its small size. The ocean outlet of the San Luis Rey River is just south of the harbor, but freshwater influx into the harbor is curtailed by a long jetty at the harbor entrance. This was the northernmost site recorded for Polyandrocarpa zorritensis in California in 1994 (Lambert \& Lambert 1998). It has persisted but is consistently slightly more abundant in fall than in spring, and occurs only at the police floats directly opposite the harbor entrance but not at the north end of the bay (back harbor), where water movement is low. Microcosmus squamiger is also abundant at the police floats but lacking in the small back harbor. Styela clava is usually more abundant in the swifter current regime near the police floats than in the back of the bay; however, $S$. plicata is usually common throughout the bay, showing its wide tolerance of variable environmental conditions as in San Diego Bay. Oceanside Harbor is notable for the complete absence of Ciona intestinalis during any of our collections dating back to 1994 , the only harbor in the SCB lacking this species (Table 1). Present only sporadically and with low density is the congeneric Ciona savignyi. This may indicate a general inhospitable environment for phlebobranchs, since Ascidia sp. and A. zara are also absent or rare here.

\section{Dana Point Harbor}

Dana Point Harbor (64 ha) is about $34 \mathrm{~km}$ northwest of Oceanside Harbor. The small San Juan Creek empties into the ocean near the harbor entrance but, as with all the other harbors, is separated from the entrance by a jetty. We monitored the Orange County Marine Science Institute floats near the harbor entrance; the rest of the marina is nearly completely enclosed by breakwaters with such low flushing that very few ascidians or other invertebrates were ever found. Symplegma reptans appeared for the first time on a long rope during the fall 1997 survey. This species appears to be filling in between our previous northern and southern records. The abundance difference between Styela plicata and S. clava reinforces the observation that $S$. clava has a preference for moving water not shared by S. plicata. 


\section{Newport Harbor}

Newport Harbor (339 ha), 23.5 km northwest of Dana Point, opens to the ocean in Corona Del Mar, and parallels the coast for nearly $6 \mathrm{~km}$ to the northwest. A long inland branch to the shallow upper back bay receives drainage from San Diego Creek as well as extensive urban runoff. The monitoring sites include large sets of floating docks at the entrance area ('fun zone'), midharbor (Lido Island), and back bay. Periodic dredging, large fluctuations in salinity and addition of pollutants from runoff cause rapid and dramatic changes in the ascidian populations (Table 3). Extensive dredging occurred in the back bay area during spring 1997, resulting in very heavy siltation and a complete die-off of the float fauna. Populations recuperated by fall but were again decimated by heavy rainfall during spring 1998. Newport Harbor is the southernmost of our sites for Molgula manhattensis.

\section{Alamitos Bay (Long Beach Marina)}

Alamitos Bay (Long Beach Marina, 20 ha) is a small, artificially created pleasure craft harbor about $26 \mathrm{~km}$ northwest of the entrance to Newport Bay. The narrow entrance channel, separated from the San Gabriel River by a rock jetty, opens into several boat basins, including the long, narrow, blind-ended Spinnaker Cove at the northern terminus of the bay. Polyandrocarpa zorritensis, which in our previous surveys was found no further north than Oceanside Harbor, appeared in the fall of 1997 in the inner harbor. Here water movement is less than at the entrance, where mussels and barnacles dominate and ascidians are scarce. Ascidia zara, though common in many other harbors, was also collected here for the first time during fall 1997. The Spinnaker Cove site, added in fall 1997, is the northernmost record for Styela canopus.

\section{Long Beach/Los Angeles Harbors}

This complex harbor system, one of the largest in the world in terms of shipping volume (1999 annual report, Port of Long Beach), extends from Long Beach to Los Angeles. It encompasses 3156 ha and opens to the sea at the Los Angeles end nearly $14 \mathrm{~km}$ west of the entrance to Alamitos Harbor. The Los Angeles River, a major drainage system, though adjacent to the harbors, is well separated by breakwaters and fill. The various channels are considered a single harbor in oceanographic terms (Anderson et al. 1993); all are periodically dredged. Water movement and ascidian abundance are higher at Impound Marina, located on the narrow Cerritos Channel, than at Watchorn Marina situated at the wide entrance to the bay system. A major container ship depot (Matson) is located adjacent to Impound Marina, with ships arriving frequently from Japanese and other Asian ports. Polyandrocarpa zorritensis appeared for the first time in the spring 1998 survey of Impound Marina. Symplegma reptans, which is absent from Alamitos Bay, appeared at Impound Marina in spring 1997 and increased in abundance during the following year but was absent in the fall 2000 survey. In this harbor system Ciona intestinalis and Styela plicata are often more abundant than C. savignyi and Styela clava.

\section{King Harbor}

This small $(<10 \mathrm{ha})$, artificially created, primarily pleasure boat harbor in Redondo Beach is adjacent to a power plant but has its own short channel opening to the ocean without input from the power plant. It is about $17 \mathrm{~km}$ northwest of Los Angeles Harbor. Polyandrocarpa zorritensis appeared for the first time in the fall 1997 survey, as it did in several Long Beach and Los Angeles harbors. This is the northernmost record for this species in California. Ciona intestinalis is much more abundant than C. savignyi. Microcosmus squamiger and $P$. zorritensis were recorded only from near the ends of our $5 \mathrm{~m}$ ropes, never from floats.

\section{Marina Del Rey}

This harbor (144 ha) is about $15 \mathrm{~km}$ northwest of King Harbor and comprises an artificially created grid of branched waterways at the inner end of a curved dredged channel that opens to the ocean. The small Ballona Creek parallels the entrance channel but is separated from it by a rock jetty. Because of very low water movement at the back of the harbor, only 1 study site was used, closest to the entrance. This harbor seems to have an optimal environment for Styela plicata, which is abundant throughout the year; $S$. clava was not recorded prior to 2000. Our ropes, which had been submerged since 1996, were completely covered by multiple layers of $S$. plicata in 2000 .

\section{Port Hueneme}

Port Hueneme Harbor (100 ha), about 80 km northwest of Marina Del Rey, is another site artificially created by dredging wetlands to create moorage for a mixture of pleasure and commercial craft. The harbor opens to the south; a long narrow entrance channel 
splits into 3 parallel branches with extremely low water movement at the north end. Jack's Landing is the closest marina to the entrance; Anacapa Isle Marina is about one-third of the distance to the back of the bay and also has good water movement. Styela clava is more abundant at Jack's Landing than at Anacapa Isle, while the opposite is true for S. plicata (Table 3).

\section{Ventura Harbor}

Ventura Harbor (ca. $66 \mathrm{ha}$ ) is approximately $21 \mathrm{~km}$ northwest of Port Hueneme. It is divided into a northern and southern branch which receive no major rivers or creeks but are subjected to residential runoff. Styela clava and S. plicata are the dominant ascidian foulers here. Microcosmus squamiger appears consistently but is rare; this is at present its northernmost record in California.

\section{Santa Barbara Harbor}

This is the northernmost of our study sites, about $39 \mathrm{~km}$ northwest of Ventura Harbor and $78 \mathrm{~km}$ south of Point Conception, the northern limit of the SCB. It houses a mixture of pleasure and fishing craft and has a wide and abrupt entrance to the ocean with virtually no entrance channel but a long breakwater guarding the entrance. It is among the smallest of the harbors in the Bight (ca. 35 ha). Santa Barbara Harbor is notable for the absence of 5 species present in San Diego Bay, the southernmost harbor in this study. Styela clava and S. plicata are both common or abundant and usually present in about equal numbers.

\section{DISCUSSION}

\section{Persistence of introduced species}

The introduced ascidians reported in this paper are highly persistent; some have remained successful for many decades, and the newest arrivals appear to be spreading. According to Williamson (1996), most introductions fail to become established in their new habitats. If this is true in southern California, these harbors must experience the arrival of many more species than recorded, as well as multiple introductions of the species that have thrived. This does not mean that they all can be found at any single point in time. There are wide seasonal fluctuations in population densities, but refuge populations not accessible by our sampling methods (probably in deeper regions of the bays) undoubtedly survive the winter die-offs by avoiding the lowered surface salinity during periods of heavy rainfall and provide breeding stock for the rapid recolonization that occurs during spring and summer. An indication of better survival at depths greater than marina float bottoms is that in a number of instances the only spring records for some species at certain locations were at the end of the $5 \mathrm{~m}$ ropes, as e.g. during spring 1997 many small Styela plicata at Coronado Yacht Club in San Diego Bay, Polyandrocarpa zorritensis at Quivera Basin in Mission Bay, and Microcosmus squamiger at Port Hueneme and King Harbor. The large numbers of boats with fouled hulls, especially small pleasure craft, that move from one harbor to another undoubtedly provide new breeding stock to recolonize denuded surfaces and also enhance gene flow between harbors. Only genetic data will elucidate this possibility.

The first species to be recognized as nonindigenous in southern California was Ciona intestinalis, already abundant by 1917 . The same description of its habits that Ritter \& Forsyth (1917) gave applies equally today: 'The perfect identity [with Mediterranean specimens] and the fact that the species appears to be distributionally restricted in this region almost entirely to localities frequented by ships, leads one to wonder if it is not an immigrant to these parts. This surmise is the more warranted by the habits of the animal, it being especially given to clinging to the under sides of floats, buoys, and the like. In these situations it flourishes most luxuriantly and occurs in enormous numbers.'

Ascidia sp., first detected in 1983, was always more abundant in San Diego and Mission Bays than further north. After fall 1997 it underwent a striking decrease in abundance, virtually disappearing in all harbors north of San Diego, where it remained common in 2000. We suspect that the return of cooler waters may have been a contributing factor in its range contraction, but since it remains common at several sites it can easily expand its distribution when environmental conditions are favorable.

\section{New arrivals and northward spread of previously introduced species}

Southern California harbors now contain mostly introduced species of ascidians, several of which were first detected during the 1990s. The latest introduction is Botrylloides perspicuum (not included in Lambert \& Lambert 1998), a widespread species in the Indo-west Pacific and Australia (Kott 1985, Monniot \& Monniot 1996, 2001) and recently reported from Belize (Goodbody 2000). It was first encountered a few $\mathrm{km}$ from the 
Naval Station in south San Diego Bay in the fall of 1997, suggesting that it may have traveled either as hull or sea chest fouling or on particulate matter in ballast water with a naval vessel, but it could have come in on any of the numerous cargo ships that arrive frequently from the west Pacific. This fulfills our prediction (Lambert \& Lambert 1998) that new introductions into southern California harbors will continue. In spring 1998, B. perspicuum was also found closer to the entrance of San Diego Bay on Shelter Island as well as in Mission Bay. By fall 2000 it was still confined to these 2 bays and had not spread northward but was more widespread in the 2 bays, and the biomass (number and size of colonies) was much larger. Its spread within the 2 bays is a result of larval transport by tidal currents around the bay or passive transport of adult larva-containing colonies between marinas in the bay by hull-fouled pleasure craft. Like all colonial species, the larvae are incubated within the adult sessile colony and released when competent to settle; their larval life can be as short as a few minutes but is usually a few hours (Grosberg \& Quinn 1986). Thus transport as free-swimming larvae in ballast water over long distances is highly unlikely, but ballast often contains floating bark chips, seaweed and other particulates that could have sessile organisms on them. Freighters and naval craft do not enter Mission Bay, but there is considerable small boat traffic between the 2 San Diego County bays. While B. perspicuum had not spread beyond these bays by August 2000, we predict that it might appear in a few Orange or Los Angeles County bays but be temperature-limited in its northward establishment.

Polyandrocarpa zorritensis has now spread into Alamitos Bay, Impound Marina and King Harbor from its more southern previous records. This confirms our prediction that this species would continue to spread northward (Lambert \& Lambert 1998) based on its wide environmental tolerances in the northern Mediterranean (Brunetti 1978-1979). The Japanese species Symplegma reptans was recorded in Dana Harbor for the first time in fall 1997, though it had previously been found both north and south of this location; this implies a probable local spread by fouled boat hulls. Based upon its abundance and widespread distribution in Japan, an indication of high tolerance to environmental variability, we predict that it will spread to other California ports. Styela canopus, limited to San Diego Bay prior to the 1990s, now occurs north to Alamitos Bay though never in large numbers. This is an interesting range extension and may indicate a potential for further northward movement of this ascidian within the Southern California Bight. This species is common on the east coast of the US at much higher latitudes than on the west coast (Van Name 1945). This may be due to the warming influence of the Gulf Stream on northern waters in the Atlantic, while the Pacific coast is cooled by the Humboldt Current, whose effects would be somewhat diminished during El Niño years. In addition there has been a small but significant increase in inshore water temperature in California over the past $60 \mathrm{yr}$, which has resulted in the northward range extension of a number of marine invertebrates (Sagarin et al. 1999).

The Japanese species Botrylloides violaceus (Saito et al. 1981, Mukai et al. 1987), among the most widespread and abundant of the nonindigenous species in the $\mathrm{SCB}$, is now known to occur in large numbers from Ensenada to Alaska on the West Coast (Mills et al. 2000, Lambert \& Sanamyan 2001, present study) and from the Canadian maritime provinces to at least as far south as Rhode Island on the East Coast (G. Lambert unpubl. obs.). Tokioka (1953) reported it as the commonest fouling botryllid ascidian in Japan. It appeared in Venice Lagoon, Italy, in the 1990s (Zaniolo et al.1998) and is now in the Netherlands (A. Gittenberger pers. comm., identification confirmed by G. Lambert). It was apparently first recorded in southern California in the 1970s by Fay \& Vallee (1979), who briefly mentioned a botryllid with an unusual larva but did not identify the species. It was common in Monterey Bay in 1989 (Boyd et al. 1990). Because it has often been confused with $B$. diegensis, the actual time of its arrival, mode of travel and probable origin remain unknown (Table 2). Van Name (1945) lists so many color variants for $B$. diegensis in southern California, including many monocolored colonies, that some of these may have been $B$. violaceus descended from west Pacific colonies introduced when transPacific shipping increased dramatically during the first half of the 20th century. Unfortunately Van Name did not describe the larvae for any of the colonies. All monocolored colonies of Botrylloides now in southern California are $B$. violaceus, and all bicolor colonies are B. diegensis (G. Lambert unpubl. obs.).

Styela clava is abundant in many harbors on the northeast coast of the US and is spreading northward into the Canadian provinces of Prince Edward Island and New Brunswick (G. Lambert unpubl. obs., N. MacNair pers. comm.). It has persisted in several harbors in Washington state and on Vancouver Island for at least $10 \mathrm{yr}$ (Lambert \& Lambert 1998, Mills et al. 2000) and is now in most major temperate harbors in the entire world, including the northwest Pacific (Sanamyan 2000).

Molgula manhattensis has been abundant in San Francisco Bay since the late 1940s (Cohen \& Carlton 1995) though its presence in southern California was not known until 1984 (Table 2). It is now common in the US Pacific Northwest (Mills et al. 2000). On the US 
East Coast this species occurs from Maine to the Gulf of Mexico (Van Name 1945, Plough 1978).

Ciona savignyi, originally described from Japan, is now abundant at most of our study sites (Tables $1 \& 3$ ) as well as San Francisco Bay (Cohen \& Carlton 1995) and Puget Sound, Washington (Mills et al. 2000). It is not known whether the Puget Sound C. savignyi derives from California or other introductions, or are southern extensions of the indigenous, more northerly subtidal populations of British Columbia (Hoshino \& Nishikawa 1985, G. Lambert unpubl. obs.).

As compared to San Francisco Bay with 8 species of introduced ascidians (Cohen \& Carlton 1995, 1998), Puget Sound with 5 (Cohen et al. 1998, Mills et al. 2000) and Alaska with only a single species (Lambert \& Sanamyan 2001), the SCB with 14 is unusually rich in nonindigenous ascidians. Other nonindigenous taxa show similar latitudinal trends (Cohen et al. 1998). Future surveys are needed to track continued changes in these distributions.

\section{Suite of invasive ascidian species}

In a study of the Mediterranean ascidians in Algeciras Harbor in southern Spain, Naranjo et al. (1996) categorized them into 3 groups based on their distribution within the harbor. Ciona intestinalis, Microcosmos squamiger, and Styela plicata, all nonindigenous to this area (though the authors did not distinguish native from introduced), were characterized as capable of living on both man-made and natural substrates; and Botryllus schlosseri as tolerant of extreme pollution in man-made environments. We find this same group of species, all nonindigenous, in most SCB harbors in the same types of habitats and environmental conditions and consider the former 3 species as similar to $B$. schlosseri in their ability to survive in highly polluted harbor waters. C. savignyi and Botrylloides violaceus are also in most SCB harbors; we predict that they will become abundant in many Mediterranean harbors as the latter already is in Venice Lagoon (Zaniolo et al. 1998). All of the successful invasive species are capable of surviving under what would appear to be very adverse circumstances, including pollution from sewage, land runoff, and heavy metals, and competition from other harbor species, and periods of lowered salinity.

\section{Seasonal changes}

Usually the spring surveys were done in April, and the fall ones in late September or October. Had we added a winter survey, or carried out the spring sur- veys 2 mo earlier, closer in time to the winter rainy season, there may have been a higher seasonal difference in species abundance. However, in our almost $30 \mathrm{yr}$ experience of examining the southern California float populations of ascidians we have seen this pattern repeated many times, of huge die-offs following heavy rain, followed by rapid recolonization. An unusually large die-off followed heavy rain in March 1938 and was documented in detail for Newport Bay by MacGinitie (1939). He found a sharp halocline at about $2.2 \mathrm{~m}$ with all ascidians and many other invertebrates killed above this line but no effect below it: 'Prior to the storm Ciona intestinalis and Styela barnharti [=S. clava $]$ hung in solid masses from the under side of many of the floats and on the pilings ... Following the storm not a single Ciona or Styela could be found ... However, those ... below a depth of 6 feet 9 inches [ca. $2.06 \mathrm{~m}$ ] were unharmed, and they were sufficiently numerous to repopulate the floats with Ciona, so that within 3 mo following the storm the floats were covered as thickly as before with Cionas, which, although they had not yet reached their maximum size, were, nevertheless, sexually mature.' We did not compare size between seasons, but spring surveys often revealed large numbers of small animals of a majority of the species, which apparently had settled 4 to $8 \mathrm{wk}$ previously. The parent populations of these juveniles could not have come from the floats. This supports our hypothesis that any float populations killed off by winter rains would quickly re-form in the spring, most likely from deeper populations. We found high survivorship through the winter of animals on the lower regions of our $5 \mathrm{~m}$ ropes installed at the marinas; in some instances these were the only spring specimens of some species that could be found. The apparent lack of differential seasonality in some species (as determined by pooling the density numbers of a given species by season over all sites) as well as the presence of all sizes of animals throughout the year (except after massive die-offs) implies that reproduction occurs throughout the year in southern California. We found ripe gametes in most of the solitary species throughout the year. A long breeding season is one of the optimal strategies exhibited by many nonindigenous species (Williamson 1996).

\section{Artificial substrates as sites for invasive species}

Harbors abound with man-made structures that provide ample available surface and a unique type of environment as potential sites for the settlement and growth of marine organisms of all sorts, and these surfaces usually become rapidly covered with a variety of marine organisms. The complete explanation for this 
extreme coverage of man-made structures is presently unknown but well documented especially for fouling organisms (Connell 2001, Holloway \& Connell 2002). Their distribution may also be partially the result of which species were reproductive at the time the substrate was submerged (Hurlbut 1991). Other factors are hydrographic features of the harbors and the arrival of exotic species. Surprisingly, though, only certain taxa occur on such habitats in harbors (Lambert \& Lambert 1998, Connell \& Glasby 1999, Glasby \& Connell 1999, Connell 2000). Communities outside harbors in more natural habitats often have quite different species composition than those inhabiting man-made substrates within the harbors. Natural habitats offer relatively stable substrates, which develop through succession a complex, highly diverse community relatively resistant to invasion by nonindigenous organisms (see Stachowicz et al. 1999 for review). In contrast, available habitat is provided whenever an artificial substrate is submerged or cleaned and can be colonized by whichever larvae are present at the time of disturbance. Recently it has been shown in a careful comparison between harbor and reef ascidians in Guam that the harbor structures are populated by a relatively few species of nonindigenous ascidians, many of which are widespread throughout the south Pacific. This group is almost completely absent from the adjoining natural reef assemblages with their much higher diversity of native species (Lambert 2003). Similarly, Fay \& Johnson (1971) found 40 species total in southern California but only 14 species on harbor floats and pilings. Ciona intestinalis, Styela clava and S. plicata, all nonindigenous, were found exclusively on floats and pilings and not on any natural surfaces. At least 42 ascidians occur intertidally in northern California but mostly outside harbors (Abbott 1975); only 14 species were recorded from San Francisco Bay in 1997 (G. Lambert unpubl. obs.). We fully agree that 'A great deal more research is needed to understand fully the consequences of adding new habitats to the marine environment' (Glasby \& Connell 1999), a statement especially relevant to the establishment of artificial reefs (J. Culbertson pers. comm.).

\section{Ecological factors and ascidian distribution}

In Norwegian fjords salinity is important in the distribution of indigenous ascidian species (Dybern 1969) and temperature may be a limiting factor in the distribution of Mytilus species on both sides of the Pacific (Suchaneck et al. 1997). Temperature and salinity measurements were made during most of our surveys, though they give only 'snapshots' of temperature and salinity and are not a suitable substitution for continu- ous records of these parameters. The mean temperature of the spring surveys was $18.23^{\circ} \mathrm{C}$ (range 13.00 to $22.00^{\circ} \mathrm{C}$ ) and the fall surveys $20.51^{\circ} \mathrm{C}$ (range 13.50 to $27.00^{\circ} \mathrm{C}$ ), the difference being statistically significant. The warmest temperatures occurred during our fall 1997 survey, with surface readings of over $27^{\circ} \mathrm{C}$ at several sites. Interestingly, this coincided with the first report of Botrylloides perspicuum in southern California and the first record of Polyandrocarpa zorritensis north of Oceanside Harbor (Long Beach). All of the introduced species survived the peak temperatures. Ascidia sp. suffered a decline north of Los Angeles during the falling temperatures seen in 1998 but an increase in San Diego and Mission Bays in 2000, when again warmer temperatures were recorded. This substantiates our prediction for this warm-water species (Lambert \& Lambert 1998).

The mean salinity values were $32.71 \%$ (range 26 to $35 \%$ ) for spring and $33.47 \%$ (range 24 to $37 \%$ ) for fall. While these salinity means are not statistically different, they mask the very large short-term changes in surface salinity that occurred periodically due to heavy winter rains, which in the most extreme cases caused a complete die-off of all ascidians on the uppermost $0.5 \mathrm{~m}$ submerged sections of the floating docks. The lower portions of the $5 \mathrm{~m}$ ropes installed at all the marinas had high survivorship of ascidians during all rainy periods, indicating that the lowered salinity affected only the top meter or so. Subsequent rapid recolonization of these surfaces is presumed to be the result of larval recruitment from surviving populations at nearby deeper sites, such as on rocks or at the bottoms of pilings.

The distribution of ascidians in natural habitats has also been ascribed to water movement (Fay \& Johnson 1971, Hernandez-Zanuy \& Carballo 2001), and we see some evidence of this in our study. In San Diego Bay Microcosmus squamiger and Styela canopus are particularly abundant in the southern part of the bay, where there is less water flow than nearer the entrance. Species generally more abundant closer to the opening of the bay and higher water movement are Ascidia zara, S. clava, S. plicata and Polyandrocarpa zorritensis. Greater overall abundance of ascidians at Impound Marina than at Watchorn also indicates that the rate of water movement may be important in the distribution of ascidians. The nearly equal density of $S$. clava and S. plicata in Santa Barbara Harbor suggests a medium current regime. The differences in relative abundance of $S$. canopus and $S$. plicata within regions of San Diego Bay suggest that swifter currents could favor $S$. plicata over $S$. canopus as the $S$. plicata greatly outnumber $S$. canopus closer to the entrance of the bay while both species are more equal in the calmer back bay (Table 3). Where it occurs, Molgula manhattensis is most abundant in quiet waters at the backs of harbors. 
Bacteria from anthropogenic activities have apparently led to huge increases in the density of a Caribbean ascidian whose spread parallels bacterial density (Bak et al. 1996). The human population adjacent to the SCB generates a very high volume of bacteria, which flows to the sea via storm drains and the sewage treatment plants. These release bacteria several hundred meters offshore, but nevertheless the impact of bacteria on inshore waters is tremendous. Since ascidians and other filter-feeding invertebrates are very efficient feeders on nanoplankton, the density of bacteria must have a profound effect upon the density of these organisms. We have scrutinized the abundance data of bacteria within Los Angeles/Long Beach Harbor, San Diego Bay and Mission Bay. Bacteria are very abundant at all these sites, though it was not possible to make any direct correlation between bacterial abundance and the differential density of ascidians.

The harbors of the SCB vary in size from only a few hectares to over 4000 ha. The 3 largest are San Diego Bay (4441 ha), Long Beach/Los Angeles Harbors (3156 ha) and Mission Bay (819 ha). Of the species with our maximum density rating of 4,9 have been this abundant during at least 1 survey in San Diego Bay, 6 species in Long Beach/Los Angeles Harbors and 7 species in Mission Bay. It is thus possible that harbor size may be a factor in species abundance and probably indicates that there is less fluctuation in temperature, salinity and other variables in the larger water masses.

\section{Co-existence or interspecific competition between introduced species of ascidians?}

According to Gaussian precepts no 2 congeneric species with similar requirements should be able to occupy the same niche for long (Roughgarden 1998). In our study the 2 most similar co-occurring species from a single genus are Ciona intestinalis and C. savignyi, which do not interbreed (Byrd \& Lambert 2000). Although $C$. intestinalis has been found in southern California harbors for more than half a century longer than C. savignyi, the latter is now more widespread (Table 2), though their densities are almost equivalent: median abundance for $C$. intestinalis $=1.133$ and 0.9171 for C. savignyi for the entire Bight (considered non-significant differences by a Mann-Whitney $U$-test). We have not found C. intestinalis in Oceanside Harbor, and C. savignyi has never been found in Dana Harbor, which may indicate differences in niche specifications. Perhaps C. savignyi would eventually replace $C$. intestinalis were it not that the frequently changing habitat apparently tends to stabilize their co-occurrence in nearly equal numbers.
Three species of Styela co-exist in San Diego Bay, but $S$. canopus is absent from other harbors containing large numbers of $S$. clava and $S$. plicata. It is possible that niche partitioning has occurred so that the 3 Styela species can co-exist without competing at specific sites in San Diego Bay. S. clava is a large, stalked species with siphons often $15 \mathrm{~cm}$ above the substrate; $S$. plicata is also large but without a stalk; its siphons would rarely be more than $10 \mathrm{~cm}$ above the substrate, while the diminutive $S$. canopus has siphons generally less than $2 \mathrm{~cm}$ above the substrate. In this case the 3 species may not be competing for the same limiting resource such as food in some harbors. A single specimen of $S$. plicata was collected from a piling in San Diego Bay in 1915 by Ritter \& Forsyth (1917); this species is now the most abundant ascidian in southern California harbors. When $S$. canopus was first recorded at J Street in south San Diego Bay in 1972, it was very abundant and there were no Microcosmus squamiger present; now $M$. squamiger greatly outnumbers it (Table 3). The most numerous solitary ascidians in the SCB were the indigenous Ascidia ceratodes and Pyura haustor in 1917 (Ritter \& Forsyth 1917). We rarely encounter $A$. ceratodes now; it appears to have been nearly completely replaced by $A$. zara. $P$. haustor is apparently completely absent from these harbors now though still abundant outside harbors (Fay \& Johnson 1971, Fay \& Vallee 1979).

\section{Systematics of introduced species}

Of the 14 introduced ascidian species, 10 are in the order Stolidobranchia and 8 of these are from the family Styelidae, including the genera Styela, Polyandrocarpa, Botryllus, Botrylloides, and Symplegma (Table 2). Also included are a single pyurid (Microcosmus) and molgulid (Molgula). Four of the invasive species are from the Order Phlebobranchia (Ciona and Ascidia). There are no invasive species from the order Aplousobranchia at our study sites; however, Diplosoma listerianum may actually be introduced (Lambert \& Lambert 1998). Nine of the 14 species are solitary and 5 are colonial. In contrast, 7 of the 9 indigenous species that also comprise the harbor fouling communities in southern California are either colonial or social (Lambert \& Lambert 1998); 5 are aplousobranchs, 2 are phlebobranchs and 2 are stolidobranchs. This indicates that stolidobranchs both solitary and colonial survive long-range anthropogenic transport better than do other types of ascidians. Having arrived, successful nonindigenous ascidians thrive in many habitats with wide variations in temperature, salinity, various pollutants and food supply. This is probably the result of a greater physiological flexibil- 
ity than the endemic species, which are presumably adapted to the specific environmental conditions that existed prior to anthropogenic activities.

Acknowledgements. We are indebted to G. Ruiz, R. Strathmann, C. Mills and J. Carlton for suggestions on the research and its interpretation. C. Clifton and R. Cressey provided extensive data on bacterial density. Y. Saito helped in the determination of Botrylloides perspicuum and B. violaceus. We are grateful to J. Carlton for sharing with us his unpublished photographs of B. violaceus in Puget Sound. E. Kellogg is thanked for providing the data on harbor sizes from satellite images. The manuscript was greatly improved by the comments of $\mathrm{C}$. Birkeland and 4 anonymous reviewers. California Sea Grant R/CZ-135 funded the work from 1996 to 1998; the data for 2000 was funded by the California State Water Resources Control Board, California Department of Fish and Game, and the National Fish and Wildlife Foundation. We are indebted to A. Lowe for help with the 1996 to 1998 surveys.

\section{LITERATURE CITED}

Abbott DP (1975) Phylum Chordata: introduction and Urochordata. In: Smith RI, Carlton JT (eds) Lights manual: intertidal invertebrates of the central California coast, 3rd edn. University of California Press, Berkeley, p 638-655

Abbott DP, Johnson JV (1972) The ascidians Styela barnharti, S. plicata, S. clava, and S. montereyensis in Californian waters. Bull South Calif Acad Sci 71:95-105

Anderson JW, Reisch DJ, Spies RB, Brady ME, Segelhorst EW (1993) Human impacts. In: Daily MD, Reish DJ, Anderson JW (eds) Ecology of the southern California Bight: a synthesis and interpretation. University of California Press, Berkeley, p 682-766

Bak RPM, Lambrechts DYM, Joenje M, Nieuwland G, Van Veghel MLJ (1996) Long-term changes on coral reefs in booming populations of a competitive colonial ascidian. Mar Ecol Prog Ser 133:303-306

Boyd HC, Weissman IL, Saito Y (1990) Morphologic and genetic verification that Monterey Botryllus and Woods Hole Botryllus are the same species. Biol Bull 178:239-250

Brunetti R (1978-1979) Polyandrocarpa zorritensis (Van Name, 1931) a colonial ascidian new to the Mediterranean record. Vie Milieu 28-29:647-652

Byrd J, Lambert CC (2000) Mechanism of the block to hybridization and selfing between the sympatric ascidians Ciona intestinalis and Ciona savignyi. Mol Reprod Dev 55: 109-116

Cohen AN, Carlton JT (1995) Nonindigenous aquatic species in a United States estuary: a case study of the biological invasions of the San Francisco Bay and delta. A report for the US Fish and Wildlife Service and National Sea Grant College Program. National Technical Information Service, Springfield, VA

Cohen AN, Carlton JT (1998) Accelerating invasion rate in a highly invaded estuary. Science 279:555-558

Cohen A, Mills C, Berry H, Wonham M and 13 others (1998) Report of the Puget Sound Expedition Sept. 8-16, 1998; a rapid assessment survey of nonindigenous species in the shallow waters of Puget Sound. Washington State Department of Natural Resources, Olympia, WA

Coma R, Ribes M, Gili JM, Hughes RN (2001) The ultimate opportunists: consumers of seston. Mar Ecol Prog Ser 219: 305-308

Connell SD (2000) Floating pontoons create novel habitats for subtidal epibiota. J Exp Mar Biol Ecol 247:183-194

Connell SD (2001) Urban structures as marine habitats: an experimental comparison of the composition and abundance of subtidal epibiota among pilings, pontoons and rocky reefs. Mar Environ Res 52:115-125

Connell SD, Glasby TM (1999) Do urban structures influence local abundance and diversity of subtidal epibiota? A case study from Sydney Harbour, Australia. Mar Environ Res 47:373-387

Dailey MD, Anderson JW, Reish DJ, Gorsline DS (1993) The California bight: background and setting. In: Dailey MD, Reish DJ, Anderson JW (eds) Ecology of the southern California Bight: a synthesis and interpretation. University of California Press, Berkeley, p 1-18

Dybern BI (1969) Distribution and ecology of ascidians in Kviturdvikpollen and Vagsbopollen on the west coast of Norway. Sarsia 37:21-40

Fay RC, Johnson JV (1971) Observations on the distribution and ecology of the littoral ascidians of the mainland coast of southern California. Bull South Calif Acad Sci 70: 114-124

Fay RC, Vallee JA (1979) A survey of the littoral and sublittoral ascidians of southern California, including the Channel Islands. Bull South Calif Acad Sci 78:122-135

Glasby TM, Connell SD (1999) Urban structures as marine habitats. Ambio 28:595-598

Goodbody I (2000) Diversity and distribution of ascidians (Tunicata) in the Pelican Cays, Belize. Atoll Res Bull 480: 302-326

Graphpad (1993) Instat 2.0 software. GraphPad Software, San Diego, CA

Grosberg RK, Quinn JF (1986) The genetic control and consequences of kin recognition by larvae of a colonial marine invertebrate. Nature 322:456-459

Hernandez-Zanuy AC, Carballo JL (2001) Distribution and abundance of ascidian assemblages in Caribbean reef zones of the Golfo de Batabano (Cuba). Coral Reefs 20: $159-162$

Holloway MG, Connell SD (2002) Why do floating structures create novel habitats for subtidal epibiota? Mar Ecol Prog Ser 235:43-52

Hoshino ZI, Nishikawa T (1985) Taxonomic studies of Ciona intestinalis (L.) and its allies. Publ Seto Mar Biol Lab 30: $61-79$

Hurlbut CJ (1991) The effects of larval abundance, settlement and juvenile mortality on the depth distribution of a colonial ascidian. J Exp Mar Biol Ecol 150:183-202

Kott P (1985) The Australian Ascidiacea. Part 1. Phlebobranchia and Stolidobranchia. Mem Queensl Mus 23: $1-440$

Lambert CC, Lambert G (1998) Nonindigenous ascidians in southern California harbors and marinas. Mar Biol 130: $675-688$

Lambert G (2001) A global overview of ascidian introductions and their possible impact on the endemic fauna. In: Sawada H, Yokosawa H, Lambert CC (eds) The biology of ascidians. Springer-Verlag, Tokyo, p 249-257

Lambert G (2003) Marine biodiversity of Guam: the Ascidiacea. Micronesica 35-36:588-597

Lambert G, Sanamyan K (2001) Distaplia alaskensis sp. nov. (Ascidiacea, Aplousobranchia) and other new ascidian records from south-central Alaska, with a redescription of Ascidia columbiana (Huntsman, 1912). Can J Zool 79: 1766-1781 
MacGinitie GE (1939) Some effects of fresh water on the fauna of a marine harbor. Am Midl Nat 21:681-686

Mills C, Cohen AN, Berry HK, Wonham MJ, and 13 others (2000) The 1998 Puget Sound Expedition: a shallow-water rapid assessment survey for nonindigenous species, with comparisons to San Francisco Bay. In: Pederson J (ed) Marine bioinvasions - proceedings of a conference January 24-27, 1999. Massachusetts Institute of Technology Sea Grant College Program, Cambridge, MA, p 130-138

Monniot F, Monniot C (1996) New collections of ascidians from the western Pacific and southeastern Asia. Micronesica 29:133-279

Monniot F, Monniot C (2001) Ascidians from the tropical western Pacific. Zoosystema 23:201-383

Morgan TH (1942) Do spermatozoa penetrate the membrane of self-inseminated eggs of Ciona and Styela? Biol Bull 82: $455-460$

Mukai H, Saito Y, Watanabe H (1987) Viviparous development in Botrylloides (compound ascidians). J Morph 193: 263-276

Naranjo SA, Carballo JL, Garcia-Gomez JC (1996) Effects of environmental stress on ascidian populations in Algeciras Bay (southern Spain). Possible marine bioindicators? Mar Ecol Prog Ser 144:119-131

Plough HH (1978) Sea squirts of the Atlantic continental shelf from Maine to Texas. Johns Hopkins University Press, Baltimore

Ritter WE, Forsyth RA (1917) Ascidians of the litteral zone of southern California. Univ Calif Publ Zool 16:439-512

Roughgarden J (1998) Primer of ecological theory. PrenticeHall, Upper Saddle River, NJ

Sagarin RD, Barry JP, Gilman SE, Baxter CH (1999) Climaterelated change in an intertidal community over short and long time scales. Ecol Monogr 69:465-490

Saito Y, Mukai H, Watanabe H (1981) Studies on Japanese

Editorial responsibility: Charles Birkeland (Contributing Editor), Honolulu, Hawaii, USA compound styelid ascidians. II. A new species of the genus Botrylloides and redescription of $B$. violaceus Oka. Publ Seto Mar Biol Lab 26:357-368

Saito Y, Shirae M, Okuyama M, Cohen S (2001) Phylogeny of botryllid ascidians. In: Sawada H, Yokosawa H, Lambert CC (eds) The biology of ascidians. Springer-Verlag, Tokyo, p 315-320

Sanamyan K (2000) Ascidians from the north-western Pacific region 7. Styelidae. Ophelia 53:67-78

Schiff KC, Allen MJ, Zeng EY, Bay SM (2000) Southern California. Mar Pollut Bull 41:76-93

Seapy RR (1974) Distribution and abundance of the epipelagic mollusk Carinaria japonica in waters off southern California. Mar Biol 24:243-250

Stachowicz JJ, Whitlatch RB, Osman RW (1999) Species diversity and invasion resistance in a marine ecosystem. Science 286:1577-1579

Stachowicz JJ, Terwin JR, Whitlatch RB, Osman RW (2002) Linking climate change and biological invasions: ocean warming facilitates nonindigenous species invasions. Proc Natl Acad Sci 99:1497-1550

Suchanek TH, Geller JB, Kreiser BR, Mitton JB (1997) Zoogeographic distribution of the sibling species Mytilus galloprovincialis and $M$. trossulus (Bivalvia: Mytilidae) and their hybrids in the north Pacific. Biol Bull 193: 187-194

Tokioka T (1953) Ascidians of Sagami Bay. Iwanami Shoten, Tokyo

Van Name WG (1945) The North and South American ascidians. Bull Am Mus Nat Hist 84:1-476

Williamson M (1996) Biological invasions. Chapman \& Hall, New York

Zaniolo G, Manni L, Brunetti R, Burighel P (1998) Brood pouch differentiation in Botrylloides violaceus, a viviparous ascidian (Tunicata). Invertebr Reprod Dev 33:11-24

Submitted: June 22, 2002; Accepted: May 25, 2003

Proofs received from author(s): September 3, 2003 